\title{
Global aphasia without hemiparesis: language profiles and lesion distribution
}

\author{
Robert E Hanlon, Warren E Lux, Alexander W Dromerick
}

\begin{abstract}
Objectives-Global aphasia without hemiparesis (GAWH) is an uncommon stroke syndrome involving receptive and expressive language impairment, without the hemiparesis typically manifested by patients with global aphasia after large left perisylvian lesions. A few cases of GAWH have been reported with conflicting conclusions regarding pathogenesis, lesion localisation, and recovery. The current study was conducted to attempt to clarify these issues.

Methods-Ten cases of GAWH were prospectively studied with language profiles and lesion analysis; five patients had multiple lesions, four patients had a single lesion, and one had a subarachnoid haemorrhage. Eight patients met criteria for cardioembolic ischaemic stroke.

Results-Cluster analysis based on acute language profiles disclosed three subtypes of patients with GAWH; these clusters persisted on follow up language assessment. Each cluster evolved into a different aphasia subtype: persistent GAWH, Wernicke's aphasia, or transcortical motor aphasia (TCM). Composite lesion analysis showed that persistent GAWH was related to lesioning of the left superior temporal gyrus. Patients with acute GAWH who evolved into TCM type aphasia had common lesioning of the left inferior frontal gyrus and adjacent subcortical white matter. Patients with acute GAWH who evolved into Wernicke's type aphasia were characterised by lesioning of the left precentral and postcentral gyri. Recovery of language was poor in all but one patient. Conclusions-Although patients with acute GAWH are similar on neurological examination, they are heterogeneous with respect to early aphasia profile, language recovery, and lesion profile.
\end{abstract}

(F Neurol Neurosurg Psychiatry 1999;66:365-369)

Keywords: aphasia; cerebrovascular disease; hemiparesis; rehabilitation

Global aphasia without hemiparesis (GAWH) is an unusual stroke syndrome of particular interest to both aphasiologists and motor control theorists, because of the dissociation of language and motor functions and the absence of focal neurological signs. Global language impairment typically occurs after large perisylvian lesions, with infarction of both the frontal and temporal branches of the left middle cerebral artery. Ordinarily, contralateral hemi- paresis co-occurs with global aphasia, due to the proximity of cortical language centres and motor control areas. ${ }^{1}$

Few reports of the GAWH syndrome are available and the available studies are generally limited to a few cases. ${ }^{2-9}$ None the less, these reports have generated some controversy in the literature over several issues. Firstly was whether a single lesion could cause GAWH or whether multiple lesions (in anterior and posterior language areas, but sparing the motor cortex) were required. Secondly was whether GAWH could be strongly indicative of an embolic condition, perhaps obviating the need for extensive evaluation before the initiation of treatment such as anticoagulation for an embolic source. Thirdly was whether this syndrome had a better prognosis for recovery of language function than the much more common global aphasia with hemiparesis.

We undertook the current prospective study to further define the linguistic variables of the GAWH syndrome using standard measures of language and motor function, to examine the role of embolic phenomena in its pathogenesis, to describe the patterns of cerebral lesions which generate the syndrome, and to document the recovery patterns. We chose the rehabilitation setting to exclude patients with rapidly resolving neurological deficits. We used the National Institutes of Health Stroke Scale (NIHSS) ${ }^{10}$ to objectively define the absence of hemiparesis and applied the criteria of the TOAST (Trial of Org 10172 In Acute Stroke

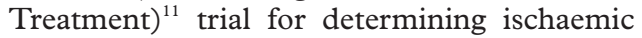
stroke subtypes. Additionally, we obtained postacute follow up language evaluations in nearly all of our subjects.

\section{Method}

All admissions to a specialty neurorehabilitation unit over an 18 month period were prospectively screened by two attending neurologists. To be included in this study, patients were required to be alert and cooperative and to have profound impairment of all language

Table 1 Demographic data, TOAST classification, ${ }^{15}$ lesion type, and $G A W H$ subtype

\begin{tabular}{llllll}
\hline Case & Age & Sex & $\begin{array}{l}\text { TOAST } \\
\text { classification }\end{array}$ & $\begin{array}{l}\text { Number } \\
\text { oflesions }\end{array}$ & $\begin{array}{l}\text { GAWH } \\
\text { subtype }\end{array}$ \\
\hline 1 & 71 & $\mathrm{M}$ & Cardioembolism & Multiple & Persistent \\
2 & 66 & $\mathrm{~F}$ & Cardioembolism & Single & NA \\
3 & 50 & $\mathrm{M}$ & Cardioembolism & Single & Persistent \\
4 & 78 & $\mathrm{~F}$ & Undetermined & Single & Wernicke \\
5 & 48 & $\mathrm{~F}$ & Other determined & Other & Wernicke \\
6 & 59 & $\mathrm{~F}$ & Cardioembolism & Multiple & TCM \\
7 & 91 & $\mathrm{~F}$ & Cardioembolism & Single & Wernicke \\
8 & 73 & $\mathrm{~F}$ & Cardioembolism & Single & TCM \\
9 & 73 & $\mathrm{~F}$ & Cardioembolism & Multiple & Persistent \\
10 & 30 & $\mathrm{M}$ & Cardioembolism & Multiple & Wernicke \\
\hline
\end{tabular}


Table 2 Severity of aphasia (AQ) and WAB scores on primary language domains during acute stage

\begin{tabular}{lccrrrr}
\hline Case & AQ & Fluency & Comp & Repeat & Naming & BNT \\
\hline 1 & 0.9 & 0 & 9 & 0 & 0 & 0 \\
2 & 3.1 & 0 & 31 & 0 & 0 & 0 \\
3 & 4.2 & 0 & 14 & 4 & 0 & 0 \\
4 & 8.9 & 3 & 9 & 0 & 0 & 0 \\
5 & 15.1 & 3 & 71 & 0 & 0 & 0 \\
6 & 29.6 & 2 & 62 & 54 & 13 & 1 \\
7 & 13.5 & 3 & 43 & 16 & 0 & 0 \\
8 & 40.2 & 2 & 76 & 56 & 47 & 11 \\
9 & 0.9 & 0 & 9 & 0 & 0 & 0 \\
10 & 18.3 & 3 & 63 & 0 & 0 & 0 \\
Normal values & 100 & 10 & 200 & 100 & 100 & $50-60$ \\
\hline AQ=Aphasia & quotient; $\quad$ WAB=western aphasia & battery; \\
BNT=Boston naming test; comp=comprehension. &
\end{tabular}

Table 3 Acute GAWH subtypes based on cluster analysis

\begin{tabular}{llll}
\hline Cluster & Case & $A Q$ & Fluency \\
\hline Cluster1 & 1 & 0.9 & 0 \\
& 2 & 3.1 & 0 \\
& 3 & 4.2 & 0 \\
Cluster 2 & 9 & 0.9 & 0 \\
& 6 & 29.6 & 2 \\
Cluster 3 & 8 & 40.2 & 2 \\
& 4 & 8.9 & 3 \\
& 5 & 15.1 & 3 \\
& 7 & 13.5 & 3 \\
\hline
\end{tabular}

$\mathrm{AQ}=$ aphasia quotient.

domains (spontaneous speech, naming, comprehension, repetition, reading, and writing) on bedside neurological examination. All subjects were required to have normal scores bilaterally on the upper extremity, hand, and lower extremity motor items of the NIHSS. ${ }^{10}$ All subjects had neuroimaging performed at least 48 hours after the onset of their neurological deficits. Using results from the clinical evaluation, aetiological subtypes of ischaemic stroke were classified by the criteria of Adams et al. ${ }^{11}$ Patients with conditions other than ischaemic stroke were listed separately.

Aphasic subtype and severity of aphasia were established on the western aphasia battery $(\mathrm{WAB})^{12}$ during the acute stage (2-3 weeks after stroke) and postacute stage (10-12 weeks after stroke). The WAB aphasia quotient (AQ) and subscales of fluency, comprehension, repetition, and naming, as well as the Boston naming test $(\mathrm{BNT})^{13}$ were used for additional language analyses. The $\mathrm{AQ}$ is a measure of severity of language impairment and is a composite score based on the oral-auditory language subscales of fluency, comprehension, repetition, and naming. A cluster analysis was conducted, based on the severity of aphasia and language profiles, to assess for the existence of subgroups within the GAWH sample. A

Table 4 Follow up language status and aphasia subtypes of 3 GAWH clusters

\begin{tabular}{llcccccl}
\hline Cluster & Case & AQ & Fluency & Comp & Repeat & Naming & \multirow{2}{*}{ Subtype } \\
\hline Cluster 1 & 1 & 0.9 & 0 & 12 & 0 & 0 & Persistent \\
& 2 & NA & NA & NA & NA & NA & NA \\
& 3 & 10.0 & 1 & 24 & 8 & 0 & Persistent \\
& 9 & 1.7 & 1 & 14 & 0 & 0 & Persistent \\
Cluster 2 & 6 & 44.4 & 43 & 100 & 74 & 28 & TCM \\
& 8 & 51.7 & 7 & 103 & 70 & 57 & TCM \\
Cluster 3 & 4 & 20.6 & & 46 & 0 & 0 & Wernicke \\
& 5 & 49.3 & 78 & 129 & 42 & 20 & Wernicke \\
& 7 & 45.5 & 5 & 53 & 52 & 19 & Wernicke \\
& 10 & 20.9 & & 109 & 0 & 0 & Wernicke \\
\hline
\end{tabular}

$\mathrm{AQ}=$ Aphasia quotient.
Kruskal-Wallis analysis of variance (ANOVA) was conducted on all language variables to assess for differences among the established subgroups. Subsequently, pairwise comparisons were completed using Mann-Whitney $U$ tests.

Lesion analysis was performed using the template method of Damasio and Damasio. ${ }^{14}$ Lesion contour mapping was performed by outlining lesions on scanned images from the atlas of Matsui and Hirano. ${ }^{15}$ One patient had only right hemispheric lesions and lesions were transposed to the left hemisphere for analysis. Images were then superimposed on a Macintosh computer using the public domain program NIH Image (available on the Internet at http://rsb.info.nih.gov/nih-image).

All case reports discovered in a review of the English language literature of GAWH due to cerebrovascular disease were examined and the TOAST criteria were applied to each case as possible; "probable" and "possible" designations were pooled for the cardioembolic and large artery atherosclerotic categories. Patients with conditions other than ischaemic stroke were listed separately. Descriptions of subacute recovery were classified as "good" at $\geqslant 6$ weeks, "poor" at $\geqslant 6$ weeks, or "insufficient data to classify" if no description was included or if language deficits had not been described to resolve at 6 weeks or later. We chose not to include the cases of Nagaratnam et at because they were a group of patients who were much more mildly affected than our patients and the others described in the literature.

\section{Results}

Ten patients were identified; all were right handed. Table 1 displays demographic data and the stroke aetiology subtype classification. ${ }^{11}$ Eight of 10 patients met criteria for probable or possible cardioembolic stroke and only one patient had no clear aetiology. One patient had an infarction after a subarachnoid hemorrhage in the left middle cerebral artery (MCA). The initial language assessment was completed at a mean time of 17 days after stroke. All patients manifested severe global language impairment, as assessed on the WAB. Table 2 presents the WAB and BNT scores at the time of the initial language assessment.

A cluster analysis using the acute language data (table 2) showed three distinct subgroups, which were differentiated based on severity of aphasia as represented by the AQ $\left(\chi^{2}=7.90\right.$, $\mathrm{p}<0.02)$ and fluency $\left(\chi^{2}=9.0, \mathrm{p}<0.01\right)$. Cluster 1 included the most severely aphasic patients (mean $\mathrm{AQ}=2.3$ ), characterised by dense nonfluent speech with severe speech initiation deficit, marked comprehension impairment, no repetition, and grossly defective naming. Cluster 2 (mean $\mathrm{AQ}=34.9$ ) patients disclosed dense non-fluent spontaneous speech, moderate comprehension impairment, minimal capacity for naming monosyllabic high frequency nouns, and limited repetition. Cluster 3 (mean $\mathrm{AQ}=13.9$ ) showed low level dysfluent speech, marked comprehension impairment, minimal or no repetition, and severely defective naming characterised by perseverative monosyllabic 


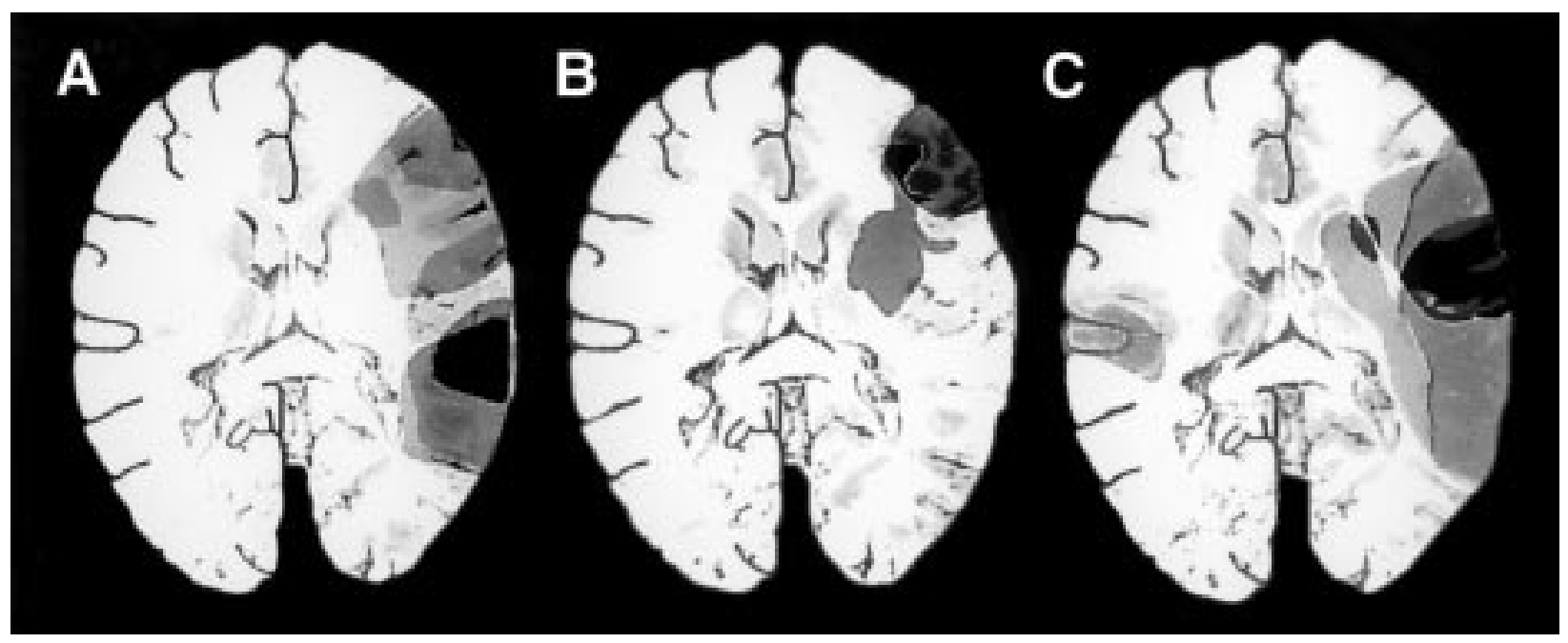

Figure 1 Composite lesion analysis of three GAWH subtypes: (A) persistent GAWH; (B) GAWH-TCM; (C) GAWH-Wernicke.

utterances. Table 3 presents the $\mathrm{AQ}$ and fluency scores of the 10 cases, according to the established clusters.

Cluster 1 and cluster 2 differed significantly with respect to fluency $(U=0 ; \mathrm{p}<0.05)$, repetition $(U=0 ; \mathrm{p}<0.05)$, and naming on both the WAB $(U=0 ; \mathrm{p}<0.05)$ and the BNT $(U=0$; $\mathrm{p}<0.05)$. Cluster 2 and cluster 3 were significantly different on the same variables as cluster 1 and cluster 2: fluency $(U=0 ; \mathrm{p}<0.05)$, repetition $(U=8 ; \mathrm{p}<0.05)$, WAB naming $(U=8$; $\mathrm{p}<0.05)$, and BNT $(U=8 ; \mathrm{p}<0.05)$. Cluster 1 and cluster 3 differed significantly for severity of aphasia (AQ) $(U=0 ; \mathrm{p}<0.05)$ and fluency $(U=0 ; \mathrm{p}<0.05)$.

Follow up language assessment completed 10-12 weeks after stroke disclosed that the three clusters established during the acute stage were characterised by differential patterns of aphasic evolvement. Patients in cluster 1 showed minimal or no change in all language domains and remained globally aphasic. Cluster 2 patients, initially characterised by the relative preservation of repetition, evolved into TCM type aphasic patients, with relative preservation of repetition, as well as notable recovery of comprehension and naming. Patients in cluster 3 showed variable language resolution, but were generally characterised by increased narrative fluency and improved auditory comprehension, with inconsistent changes in repetition and naming. All patients in cluster 3 evolved into a Wernicke's type aphasia profile, based on WAB criteria. Table 4 provides the follow up raw language scores from the WAB on individual patients within three clusters.

Composite lesion analysis showed that patients in cluster 1 , who presented with the most severe global aphasia initially, and showed minimal or no significant recovery of language functions during the first 3 months after stroke, were characterised by common lesioning of the left superior temporal gyrus, primarily involving territory of the posterior temporal and middle temporal branches of the MCA (fig A). Patients with acute GAWH who evolved into TCM type aphasic patients (cluster 2) had common lesioning of the left inferior frontal gyrus and adjacent subcortical white matter, primarily involving the territory of the precentral, central, lenticulostriate, and insular branches of the MCA (fig B). Patients with acute GAWH who evolved into Wernicke's type aphasia were characterised by lesioning of the left precentral and postcentral gyri, involving the central and posterior parietal vascular territories (fig C).

\section{Discussion}

GAWH is a striking clinical phenomenon because of the unusual dissociation between language and motor functions. Because global aphasia typically results from large perisylvian lesions, adjacent motor areas are usually affected. The absence of hemiparesis suggests that the cortical representation of motor and language function may not always follow classic descriptions, or that only unusual lesions may cause this syndrome.

Although patients with acute GAWH are similar on neurological examination, they are heterogeneous with respect to early aphasia profile, language recovery, and lesion profile. Our findings disclose three distinct subtypes of GAWH, based on acute language profiles and aphasic evolvement during the first 3 months after stroke. One subtype (persistent GAWH) presents with extremely dense global aphasia with virtually no expressive or receptive language function and is characterised by negligible recovery of language. A second subtype (GAWH-TCM) presents with acute global aphasia, characterised by limited expressive and receptive language capacity, specifically involving low level repetition and naming, and evolves into TCM type aphasia. A third subtype (GAWH-Wernicke) with moderately severe global aphasia during the acute stage evolves into Wernicke's type aphasia. Although we differentiated three subtypes of GAWH, it is important to note that classification based on a cluster analysis does not necessarily denote anatomically distinct or functionally homogeneous subtypes.

Our anatomical findings are consistent with the collective findings of previous case reports which hold that various lesion constellations, 
including both single and multiple lesions, can produce GAWH. Early reports of GAWH suggested that multiple lesions which involved anterior and posterior language areas but spared the motor cortex were necessary to cause this syndrome, and that it was highly indicative of embolic aetiology. Van Horn and Hawes $^{2}$ reported three patients with GAWH, all of whom had two lesions involving the left posterior frontal area and the left temporoparietal region. They concluded that GAWH is a sign of embolic encephalopathy, caused by two discrete lesions. Tranel et $a l^{\beta}$ described three cases, again due to double lesions involving the left frontal operculum and the left parietotemporal region. They also concluded that GAWH is produced by two discrete lesions of the left middle cerebral artery and is an indicator of embolic encephalopathy. However, later reports questioned both the necessity for multiple lesions and the role of embolism in GAWH. Ferro $^{4}$ reported two patients, each of whom had a single large infarct of the left MCA involving both Broca's and Wernicke's areas, but which spared the posterior limb of the internal capsule. Legatt et $a \bar{l}$ reported six nonhemiparetic patients with global aphasia, including one with a single infarct of the anterior branches of the left MCA, two with subarachnoid hemorrhage, and one with multiple metastases six months after presentation. Subsequently, Deleval et al ${ }^{6}$ reported two patients with GAWH caused by single prerolandic infarcts. Bogousslavsky ${ }^{7}$ described a case of GAWH secondary to a single left temporoparietal infarct and noted that two infarcts are not necessary to produce GAWH, and that the syndrome does not necessarily imply a double lesion or an embolic aetiology.

Nagaratnam et $a l^{9}$ reported 12 patients described as having GAWH. However, it is unclear how several of their patients met Boston diagnostic aphasia examination (BDAE) criteria for global aphasia because they were only mildly impaired on nearly all language functions assessed, according to scores on the aphasia severity rating scale. They reported that four cases had severe global language impairment, but "the remainder had moderate to mild abnormalities." This series seems to describe a different population from the other reports in the literature and from our own.

In addition to the different lesion profiles of the three GAWH clusters, many of our individual patients were similar to previously reported patients. Case 1 (table 1 ) had a posterior lesion involving the posterior temporal region without anterior involvement which was similar to a case reported by Bogousslavsky. ${ }^{7}$ In addition to the finding that GAWH may result from a single temporal lesion, these patients support the notion that fluency may be seriously disrupted by posterior lesions, in the absence of anterior involvement. Conversely, two patients in the present sample (case 5 from cluster 3; case 8 from cluster 2) had anterior lesions with no posterior involvement. These patients were similar to the two patients described by Deleval et al with respect to lesion site and language recovery. They provide further evidence of the notion that receptive language functions may be severely disrupted by anterior lesions, exclusively.

Such anatomical diversity is well documented in case series of the more common global aphasia with hemiparesis. Although global aphasia is commonly associated with large left perisylvian lesions involving both the anterior and posterior language areas, lesion analyses of groups of patients with global aphasia have consistently shown that the lesion constellations responsible for global language impairment are extremely variable. In a series of 37 patients with global language impairment, Vignolo $e \mathrm{al}^{16}$ found that only $22(59 \%)$ of their patients had large left perisylvian lesions involving both Broca's and Wernicke's areas. Eight $(22 \%)$ had anterior lesions sparing Wernicke's area, three $(8 \%)$ had posterior lesions sparing Broca's area, and four (11\%) had deep lesions of the insula and lenticular nucleus. Similarly, Scarpa et $a l^{17}$ reported that only 53\% of a series of 46 patients with global language impairment showed the classic anteriorposterior lesion profile, whereas $32 \%$ of their patients manifested global aphasia secondary to a deep lesion. Chronic global language impairment has also been documented after a single lesion of the left thalamus. ${ }^{18}$

Our data in conjunction with the other cases reviewed (table 5) suggest that whereas cardioembolism is the single most common aetiology of GAWH, an equal number of cases result from other conditions. The clinical evaluation of some of the prior cases reported as embolic actually showed occlusion of the internal carotid or of a cortical branch on evaluation; these cases are currently classified as large artery atherosclerotic strokes in the TOAST scheme. Although some such cases may represent stroke due to artery to artery embolisation or occult cardioembolism, other cases in this category may be atherogenic or thrombotic and thus are stroke causes which many neurologists might not currently treat with anticoagulation. Additionally, almost $20 \%$ of the cases in table 5 were due to conditions such as intracerebral haemorrhage, subarachnoid haemorrhage, or metastasis in which anticoagulation is clearly contraindicated. Thus although the finding of GAWH is suggestive of

Table 5 Summary of 27 reported cases of GAWH

\begin{tabular}{lllr}
\hline & $\begin{array}{l}\text { Previous } \\
\text { cases }\end{array}$ & $\begin{array}{l}\text { Current } \\
\text { series }\end{array}$ & Total \\
\hline Lesion type: & & & \\
$\quad$ Single ischaemic stroke & 6 & 5 & 11 \\
Multiple ischaemic stroke & 7 & 4 & 11 \\
$\quad$ Other & 4 & 1 & 5 \\
TOAST classification: & 5 & 8 & 13 \\
$\quad$ Cardioembolic & 5 & 0 & 5 \\
$\quad$ Large artery atherosclerotic & 5 & 2 & 7 \\
$\quad$ Other† & 2 & 0 & 2 \\
$\quad$ Insufficient data to classify & 2 & 1 & 7 \\
Subacute recovery: & 6 & 8 & 13 \\
$\quad$ Good & 5 & 1 & 7 \\
$\quad$ Poor & 6 & & \\
Insufficient data to classify & 6 & & \\
\hline
\end{tabular}

^Intracerebral haemorrhage one; subarachnoid haemorrhage three; metastasis one.

†TOAST undetermined classification two; intracerebral haemorrhage one; subarachnoid haemorrhage three; metastasis one 
an embolic stroke process, it is clearly not pathognomonic of embolism and these patients will require thorough evaluation for the cause of their cerebral infarction.

By contrast with previous reports, ${ }^{36}$ the patients in this study were not uniformly characterised by extensive recovery of language function. Moreover, the degree of recovery was related to the subtype of GAWH. Patients with persistent GAWH (cluster 1) showed minimal or no recovery 3 months after onset and continued to manifest severe dense global aphasia. Conversely, patients with GAWHTCM (cluster 2) disclosed notable recovery in most language domains. Finally, GAWHWernicke (cluster 3) patients disclosed significant language recovery, but only in selective language domains (fluency, comprehension). Clearly, patients with acute GAWH do not invariably experience significant language recovery. Our findings also support the utility of detailed early language assessment for this population. In our study, the first neuropsychological evaluation identified findings which were associated with those patients with residual function who went on to better long term recoveries. Such residual function can also be exploited in current rehabilitation interventions and may increase functional communication with care givers.
1 Damasio AR. Aphasia. N Engl f Med 1992;326:531-9.

2 Van Horn G, Hawes A. Global aphasia without hemiparesis: a sign of embolic encephalopathy. Neurology 1982;32:4036.

3 Tranel D, Biller J, Damasio H, et al. Global aphasia without hemiparesis. Arch Neurology 1987;44:304-8.

4 Ferro JM. Global aphasia without hemiparesis. Neurology 1983;33:1106.

5 Legatt AD, Rubin MJ, Kaplan LR, et al. Global aphasia without hemiparesis: multiple etiologies. Neurology 1987; 37:201-5.

6 Deleval J, Leonard A, Mavroudakis N, et al. Global aphasia without hemiparesis following prerolandic infarction. Neurology 1989;39:1532-5.

7 Bogousslavsky J. Global aphasia without other lateralizing signs. Arch Neurology 1988;45:143.

8 Nagaratnam N, Patston K. Global aphasia without hemiparesis. Fournal of Neurological Rehabilitation 1990;4:16972 .

9 Nagaratnam N, Barnes R, Nagaratnam S. Speech recovery following global aphasia without hemiparesis. Fournal of Neurological Rehabilitation Rehab 1996;10:115-9.

10 Brott T, Adams HP, Olinger CP, et al. Measurement of acute cerebral infarction. A clinical examination scale. Stroke 1989;20:864-70.

11 Adams HP, Bendixen BH, Kappelle J, et al. Classification of subtype of acute ischemic stroke. Definitions for use in a multicenter clinical trial. Stroke 1993;24:35-41.

12 Kertesz A. Western aphasia battery. Orlando: Grune and Stratton, 1982.

13 Kaplan E, Goodglass H, Weintraub S. Boston naming test. Philadelphia: Lea and Febiger, 1983.

14 Damasio H, Damasio AR. Lesion analysis in neuropsychology. New York: Oxford University Press, 1989.

5 Matsui T, Hirano A. An atlas of the human brain for computerized tomography. Tokyo: Igaku-Shoin, 1978.

16 Vignolo LA, Boccardi E, Caverni L. Unexpected CT-scan findings in global aphasia. Cortex 1986;22:55-69.

17 Scarpa M, Colombo A, Sorgato P, et al. The incidence of aphasia and global aphasia in left brain-damaged patients. Cortex 1987;23:331-6.

18 Kumar R, Masih AK, Pardo J. Global aphasia due to thalamic hemorrhage. A case report and review of the literature. Arch Phys Med Rehabil 1996;77:1312-5. 